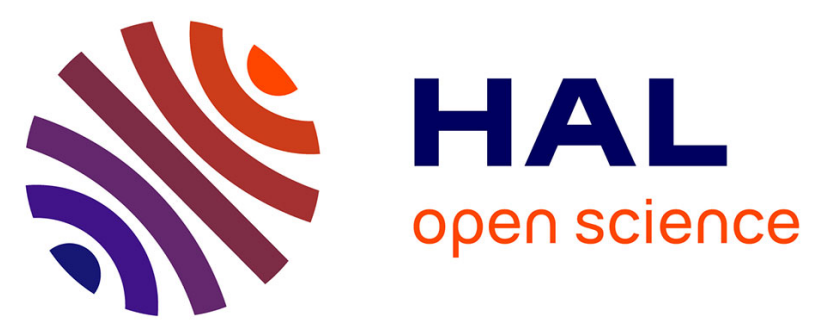

\title{
Benchmarking 2D against 3D FDTD codes for the assessment of the measurement performance of a low field side plasma position reflectometer applicable to IDTT
}

F. da Silva, E. Ricardo, J. Ferreira, J. Santos, S. Heuraux, A. Silva, T. Ribeiro, G. de Masi, O. Tudisco, R. Cavazzana, et al.

\section{To cite this version:}

F. da Silva, E. Ricardo, J. Ferreira, J. Santos, S. Heuraux, et al.. Benchmarking 2D against 3D FDTD codes for the assessment of the measurement performance of a low field side plasma position reflectometer applicable to IDTT. Journal of Instrumentation, 2022, 17, 10.1088/1748-0221/17/01/c01017 . hal-03516997

\section{HAL Id: hal-03516997 \\ https://hal.univ-lorraine.fr/hal-03516997}

Submitted on 7 Jan 2022

HAL is a multi-disciplinary open access archive for the deposit and dissemination of scientific research documents, whether they are published or not. The documents may come from teaching and research institutions in France or abroad, or from public or private research centers.
L'archive ouverte pluridisciplinaire HAL, est destinée au dépôt et à la diffusion de documents scientifiques de niveau recherche, publiés ou non, émanant des établissements d'enseignement et de recherche français ou étrangers, des laboratoires publics ou privés. 


\title{
Benchmarking 2D against 3D FDTD codes for the assessment of the measurement performance of a low field side plasma position reflectometer applicable to IDTT
}

\author{
F. da Silva, ${ }^{a},{ }^{*}$ E. Ricardo, ${ }^{a}$ J. Ferreira,${ }^{a}$ J. Santos,${ }^{a}$ S. Heuraux,${ }^{b}$ A. Silva,${ }^{a}$ T. Ribeiro,${ }^{c}$ \\ G. De Masi, ${ }^{d}$ O. Tudisco, ${ }^{e}$ R. Cavazzana ${ }^{d}$ and O. D'Arcangelo ${ }^{e}$ \\ a Instituto de Plasmas e Fusão Nuclear, Instituto Superior Técnico, \\ Universidade de Lisboa, 1049-001 Lisboa, Portugal \\ ${ }^{b}$ Institut Jean Lamour UMR 7198 CNRS-Université de Lorraine, \\ ARTEM BP 50840, F-54011 Nancy, France \\ ${ }^{c}$ Max-Planck-Institut für Plasmaphysik, \\ Garching D-85748, Germany \\ ${ }^{d}$ Consorzio RFX, 35127 Padova, Italy \\ eAssociazione EURATOM-ENEA-CR Frascati,00044 Frascati, Italy
}

E-mail:tanatos@ipfn.tecnico.ulisboa.pt

AвstRACт: O-mode reflectometry, a technique to diagnose fusion plasmas, is foreseen as a source of real-time (RT) plasma position and shape measurements for control purposes in the coming generation of machines such as DEMO. It is, thus, of paramount importance to predict the behavior and capabilities of these new reflectometry systems using synthetic diagnostics. Finitedifference time-domain (FDTD) time-dependent codes allow for a comprehensive description of reflectometry but are computationally demanding, especially when it comes to three-dimensional (3D) simulations, which requires access to High Performance Computing (HPC) facilities, making the use of two-dimensional (2D) codes much more common. It is important to understand the compromises made when using a 2D model in order to decide if it is applicable or if a 3D approach is required. This work attempts to answer this question by comparing simulations of a potential plasma position reflectometer (PPR) at the Low Field-Side (LFS) on the Italian Divertor Tokamak Test facility (IDTT) carried out using two full-wave FDTD codes, REFMULF (2D) and REFMUL3 (3D). In particular, the simulations consider one of IDTT's foreseen plasma scenarios, namely, a Single Null (SN) configuration, at the Start Of Flat-top (SOF) of the plasma current.

KeYwords: Nuclear instruments and methods for hot plasma diagnostics; Simulation methods and programs 


\section{Contents}

1 Introduction 1

2 Modelling and simulation setup $\quad 2$

$3 \quad$ Simulation and results 4

4 Conclusions $\quad 7$

\section{Introduction}

O-mode reflectometry, a technique to diagnose fusion plasmas, is foreseen as a source of real-time (RT) plasma position and shape measurements for control purposes in the coming generation of machines such as IDTT or DEMO. It is, thus, of paramount importance to predict the behaviour and capabilities of these new reflectometry systems using synthetic diagnostics (SD). Finite-Difference Time-Domain (FDTD) has become one of the mainstream numerical techniques used to simulate wave propagation in a plasma and is used to set up reflectometry synthetic diagnostics, able to offer a comprehensive view of frequency swept reflectometry, including propagation in the plasma, the emission and reception structures (antennas), the location of the system within the vacuum vessel, and to assess the signal processing techniques to be used or developed in the analysis of the results. Often, neglected, an accurate representation of the behaviour of the antennas and waveguides is paramount since they play a major role to filter and convert the reflected signal into the fundamental mode and provide an account of the electric and magnetic radiation pattern efficiency. Although FDTD is able to describe the behaviour of these diagnostics in a very complete way, is a computational demanding technique. In order to achieve an accurate description of the phase, largely below the error bars of the experimental phase measurements, the spatial discretization of the system has to be a fraction of the wavelength of the probing wave, leading to large simulation domains, specially if the simulated problem is large as is the case of the future fusion machines. To comply with Courant-Friedrichs-Lewy conditions, a small spatial discretization, implies a small temporal discretization. A large number of grid points involves large memory requirements. It also takes more time to compute the whole volume for each iteration step and since time is sampled with small time steps, the complete simulation can be costly on wallclock, specially in 3D. For these reasons, reflectometry is primary supported with 2D simulations due to the extended computational resources needed for 3D. The first tentative uses of 3D codes confirm a much more complete and close to reality description of problems. It is important to benchmark the two types of codes to assess the main differences and compromises done when using $2 \mathrm{D}$ versus $3 \mathrm{D}$. A first effort has 
been performed using pyramidal horn antenna on a monostatic and two bistatic setups with a slab plasma [1]. This work follows that and proposes an analysis of one of the tentative reflectometers for a plasma position reflectometer for IDTT, as modelled in [2], confronting a 2D and 3D SDs for the equatorial position. We used the REFMULF, 2D, and REFMUL3, 3D, codes in this work.

\section{Modelling and simulation setup}

The implementation of a SD involves emulate the hardware part of the system, including the antennas and waveguides, the access to the plasma and the vessel wall together with modelling the plasma density. For the last one we used a model based on the density profiles appearing in [3] using a fit on the extracted data based on the expression proposed in [4]. The full process to obtain the 2D poloidal maps, $n_{e}(x, y)$, is presented in [2]. For 3D density volume, $n_{e}(x, y, z)$, the 2 density map is extruded in the third dimension slab-wise, which is justified with the knowledge that the probing size in the toroidal direction $(z)$ is much smaller than the major radius of the machine. In [2] we conducted a study spanning the K, Ka, Q, V and W microwave bands, needed to probe until the separatrices of IDTT Single Null (SN) and double null scenarios. In this first 3D simulations, an ongoing work, we start with just bands K and Ka with the SN scenario, at the Start of Flattop (SOF), of the plasma current, at $T=36 \mathrm{~s}$ where a PPR is required to fulfil its main function. In figure 1 we observe the position of the PPR (gap $0^{\circ}$ ) considered for this work at the low field side equatorial plane, in machine coordinates $(R, Z)$, together with the density profile for the SN at the SOF with the different microwave bands accesses, on a length coordinate $L$. The code raw coordinates in grid points have been referenced to SI units having the origin on the wall and increasing into the vessel, a common way to proceed in reflectometry experiments. This SI code reference is used in all simulation snapshots presented for herein and its relation to the machine coordinates can be inferred on the SD showing in figure 1, left.
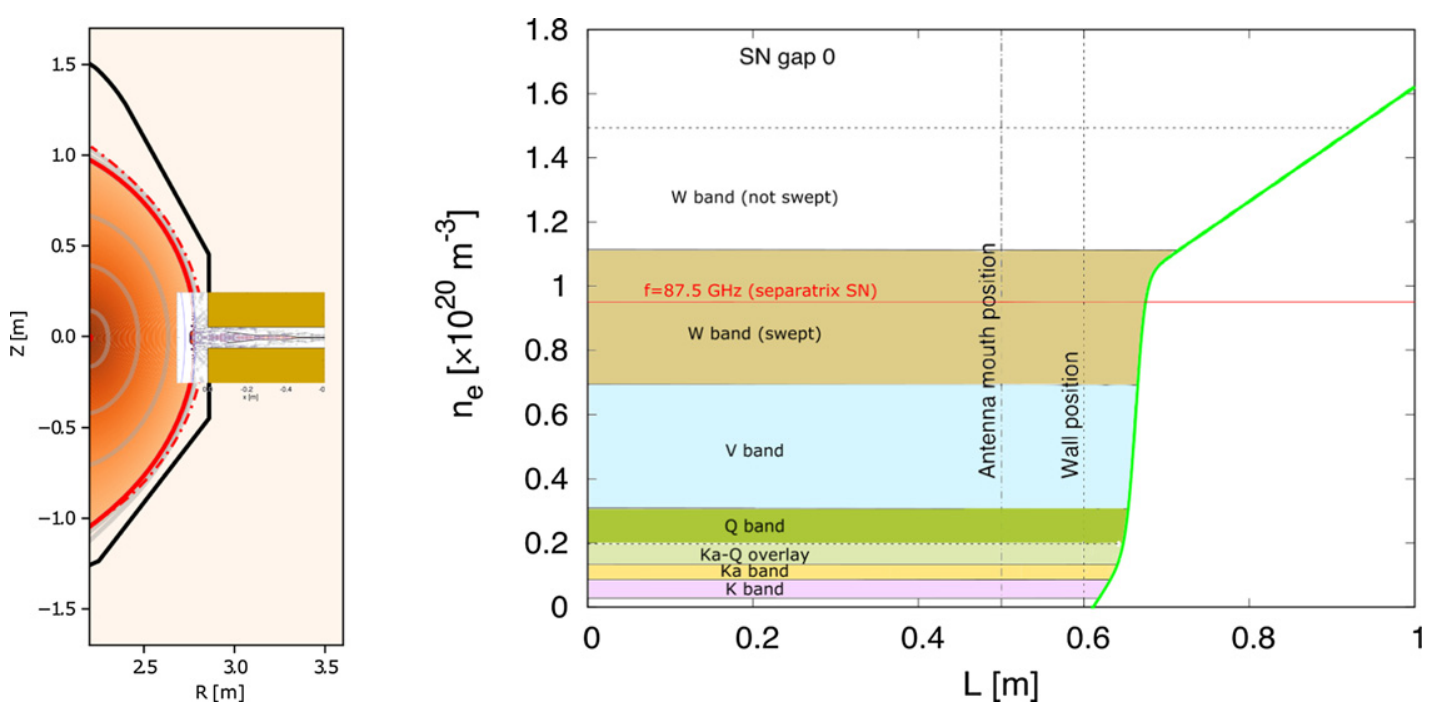

Figure 1. On the left, the representation of the IDTT vacuum vessel with gap $0^{\circ}$ put in evidence. On the right, the density profile with the different bands accesses. 
Hardware is set up from the information provided by the CAD of the inner vessel and the antennas follow one of the designs presently proposed for DEMO PPR, as seen on figure 2 - left., since a model for IDTT antennas has not yet been decided. The antennas are reassessed $10 \mathrm{~cm}$ into the first wall for thermal and erosion protection. The transmission $(\mathrm{Tx})$ and reception $(\mathrm{Rx})$ antennas are standard horns with the $H$-plane in the poloidal plane forming a bistatic configuration side-by-side in the toroidal direction. On the right column of figure 2, the 3D synthetic setup is shown and the antenna pair is seen recoiled in the cylindrical access aperture. Two poloidal planes, sectioning the Rx and Tx antennas through the $H$-planes, are marked. Collapsing these Tx and $\mathrm{Rx} H$-planes into a single 2D Tx/Rx plane is the base for the reduction to the 2D setup, detailed in [1]. This 2D counterpart appears on the left bottom. The shaded part between $[-0.4,-0.6][\mathrm{m}]$ in the simulation box emulates a termination to the antenna giving a boundary to the oversized antenna and waveguide numerically equivalent to a well behaved transmission line. On the 3D SD, the bistatic configuration separates the emitted signal from the returned one. On the 2D model, this is ensured by the use of a Unidirectional Transparent Source (UTS) [5]. The UST allows emission in the waveguide on one direction only, while permitting the weaker returning signal to pass transparently through the emission position and be detected separated from the much stronger emitted signal.

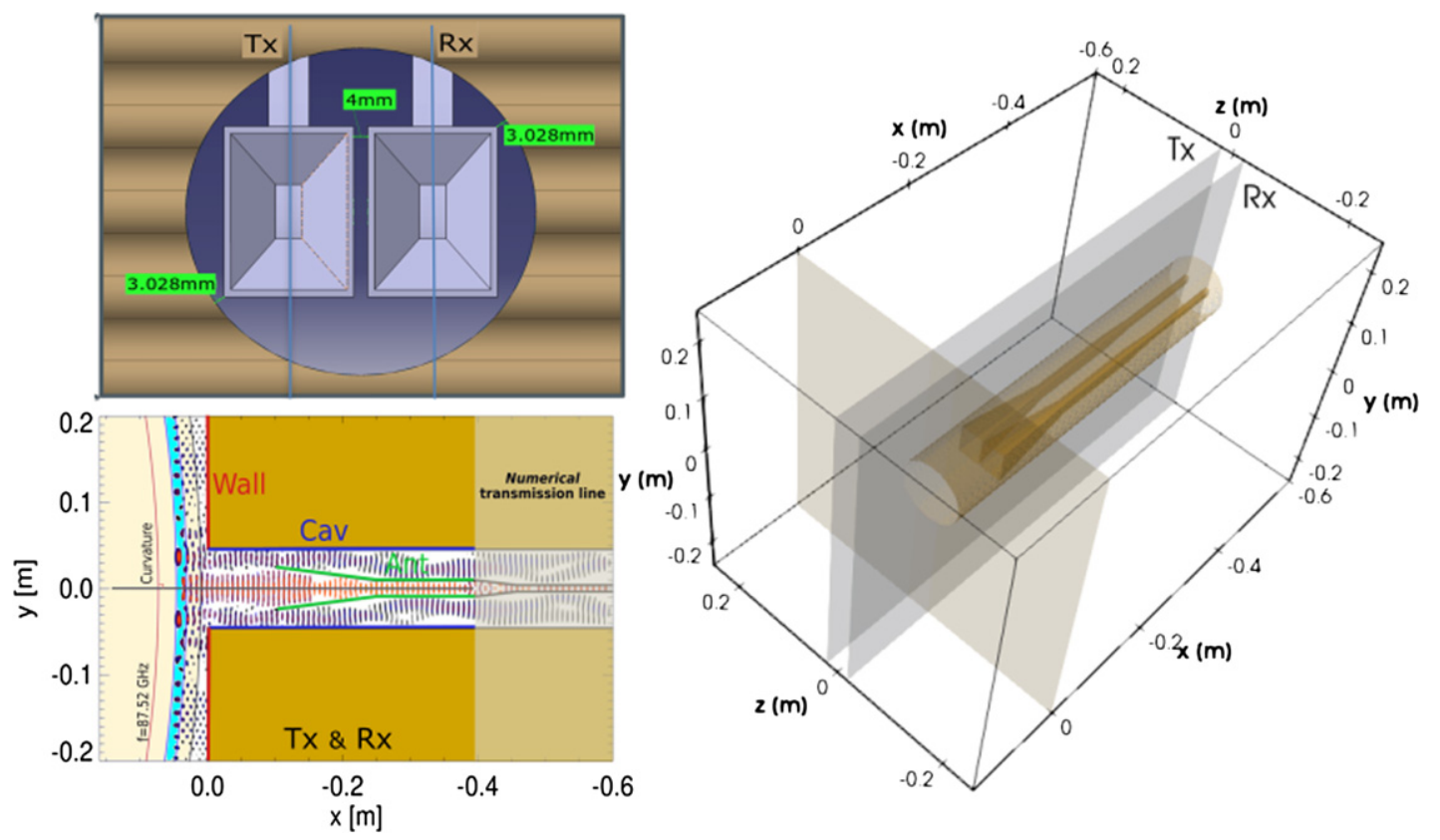

Figure 2. From the top left, in clockwise orientation, the CAD depiction of the transmission and reception antennas facing the vessel from a recessed position into a tubular access. Next the 3D SD model showing the poloidal Tx and Rx planes. Last, the 2D SD, coalescing Tx and Rx into a single plane. 
Simulations were made exciting a frequency modulated continuous waves signal covering the frequency range of each of the bands, [18-26.5 GHz] for $\mathrm{K}$ and $[26.5-40 \mathrm{GHz}]$ for Ka.

The required spatial resolution is a fraction of the vacuum wavelength $\lambda_{0}, \delta_{r}=\lambda_{0} / N$, where $N$ is the number of points per wavelength in vacuum. The higher the value of $N$, the lower the phase error committed in the simulations. For these simulations $N=20$ was used with both REFMULF and REFMUL3. This gives a point density of $1767 \mathrm{pts} / \mathrm{m}$ at the $\mathrm{K}$ band, increasing to about $2667 \mathrm{pts} / \mathrm{m}$ for the Ka. Such values conduct to grid sizes in 2D which are quite manageable for present day computer resources, $1348 \times 899$ for K and $2033 \times 1356$ for Ka bands. For both bands, simulations run for 120,000 iterations (time steps). This situation changes for 3D simulations with bands $\mathrm{K}$ and Ka reaching a considerable size, turning calculations into a high performance computing problem. For $\mathrm{K}$ band the volume grid size is $1348 \times 899 \times 899$ leading to a total count of 1, 089, 454, 948 grid points. The Ka has a volume $2033 \times 1356 \times 1356$ resulting in total of 3,738,150, 288 grid points. The number of time steps per run is the same as in $2 \mathrm{D}$. A K volume run takes $7 \mathrm{~h}$ of wallclock while the Ka band wallclock goes up to $40 \mathrm{~h}$ in Marconi Skylake partition using 64 nodes (3072 cores) with 4 MPI tasks/node (total of 256 tasks) and 12 OpenMP threads/task.

\section{Simulation and results}

On figure 3 we present a snapshot of the SD for 2D simulation while the equivalent situation for 3D appears on figure 4 . These snapshots have been taken at iteration $t_{k}=20 \times 10^{3}$. Poloidal Tx and $\mathrm{Rx} 2 \mathrm{D}$ cuts of the 3D volume appear on figures 5 and 6 , respectively. Also a 2D clip of 3D volume, on a $x-z$ plane passing through the origin of $y$ is displayed at figure 7. On the 2D SD snapshot and on the Tx plane, the electric field $E_{z}$ is the sum of the emitted and reflected field components, except for the terminal part of the 2D waveguide where the use of the UTS recovers the reflected field. On the Rx plane the emission field contribution is weaker and the relative weight of the reflected field increases. The difference between the emitted and reflected amplitudes of the signal can be viewed in figure 8 where the $E_{z}$ electric field amplitudes along the Lines Of Sight (LOSs) of the Tx and Rx are displayed. For comparison, the amplitude of the 2D Rx + Tx LOS is included.

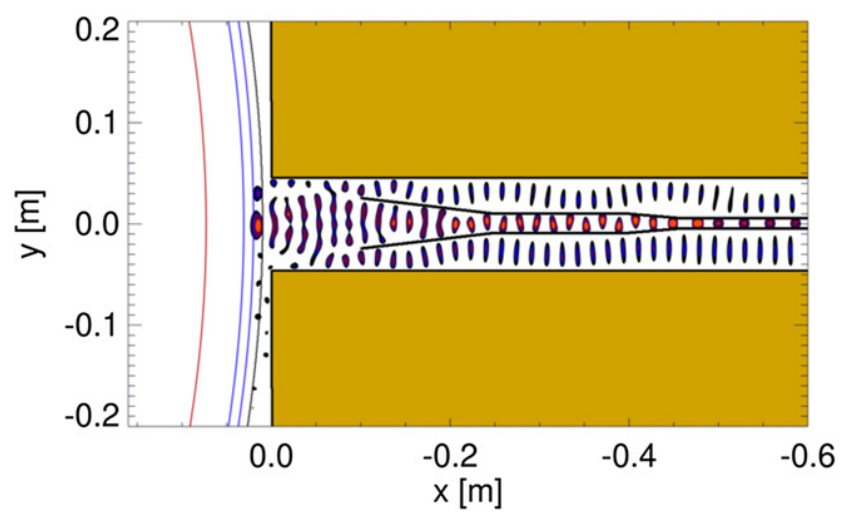

Figure 3. Snapshot of the $2 \mathrm{D} \mathrm{K}$ band simulation taken at a frequency of $f=18 \mathrm{GHz}$. 


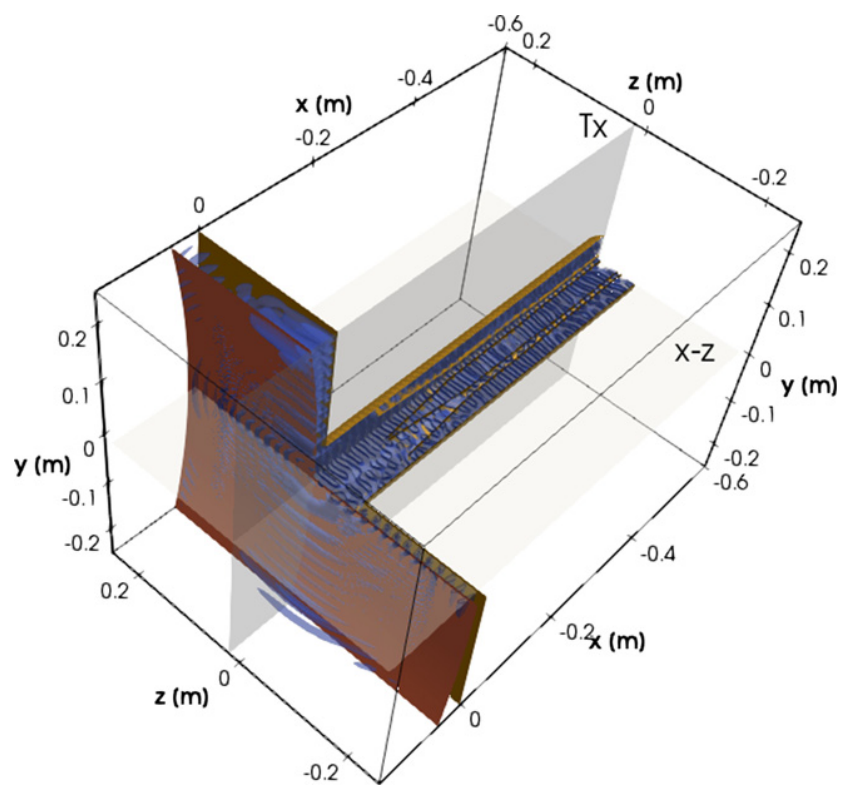

Figure 4. Snapshot of $3 \mathrm{D} \mathrm{K}$ band simulation taken at a frequency of $f=18 \mathrm{GHz}$. Part of the 3D volume has been removed to reveal the inner structure of the electric field $E_{z}$.
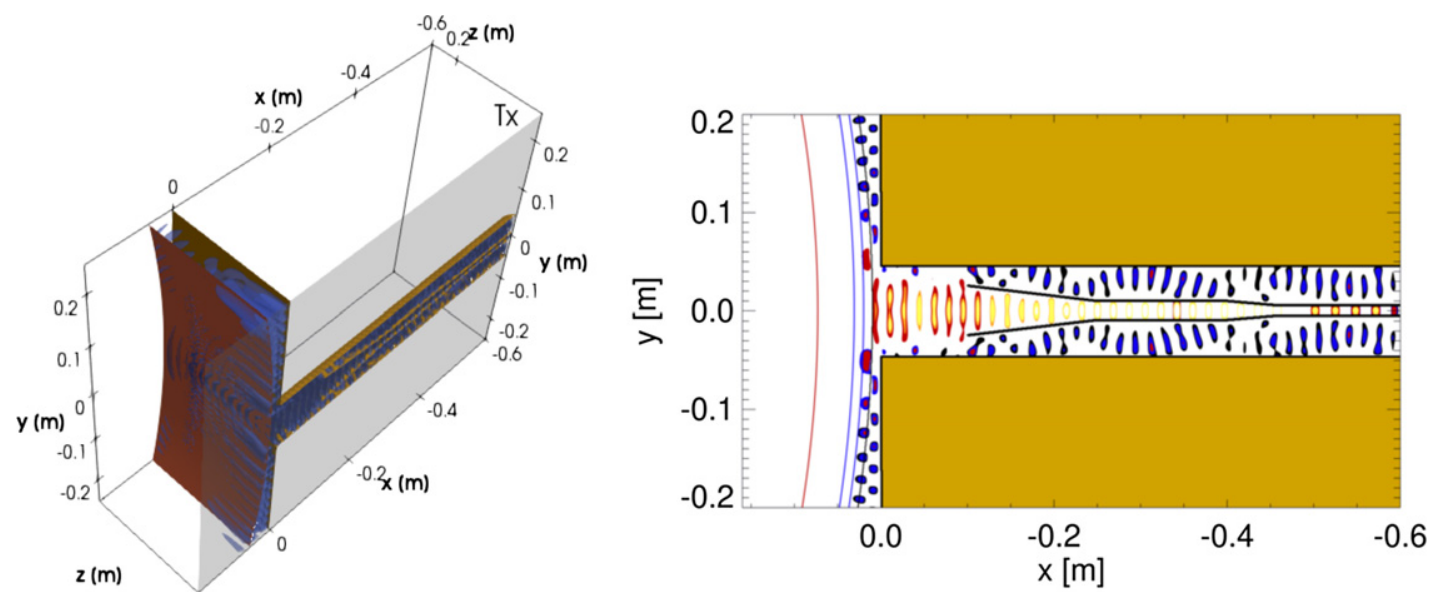

Figure 5. On the left, a clip of the 3D volume cut through the Tx plane. On the right, the 2D Tx slice plane taken from the $3 \mathrm{D}$ simulation. 

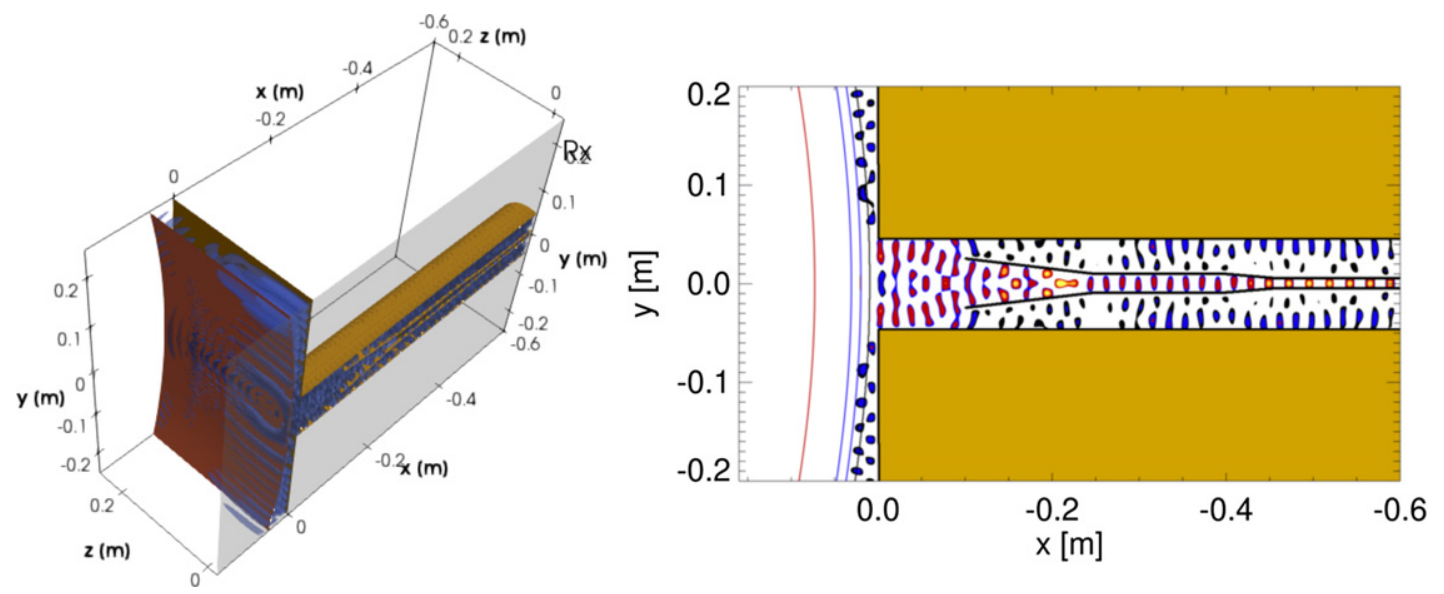

Figure 6. On the left, a clip of the 3D volume cut through the Rx plane. On the right, the 2D Rx slice plane taken from the 3D simulation.
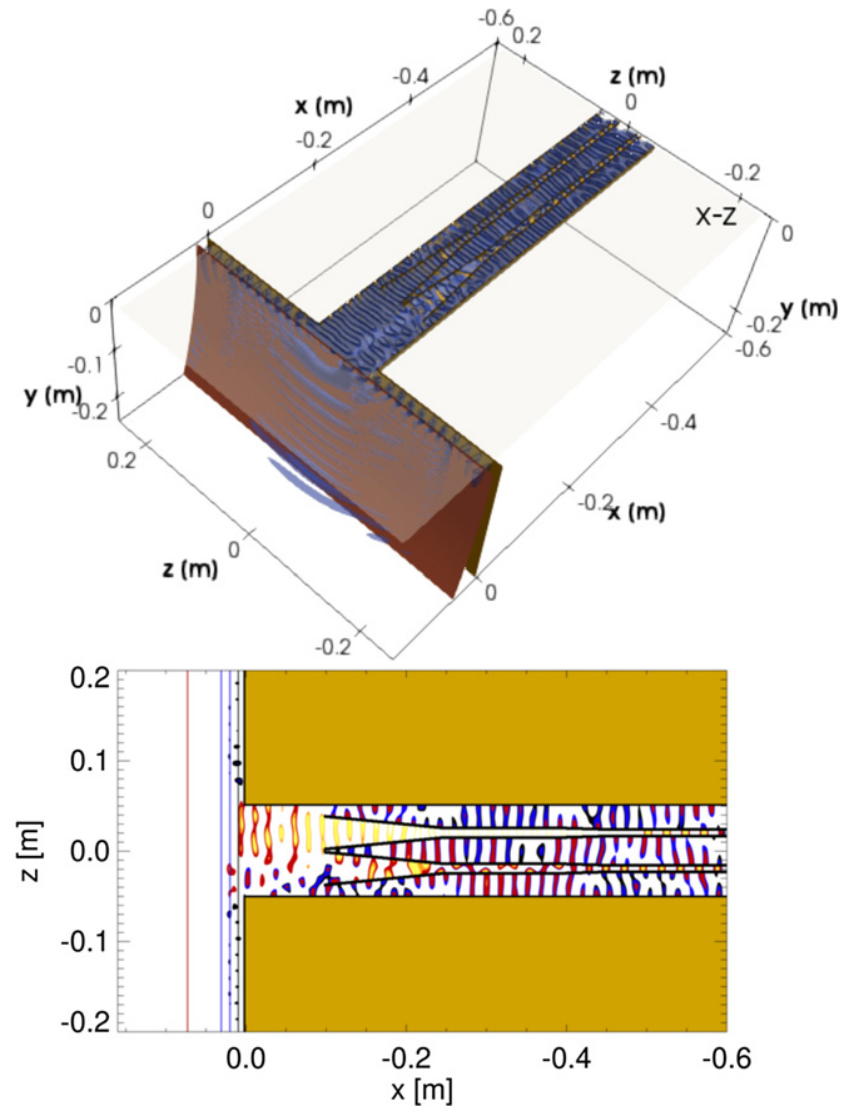

Figure 7. On the top, a clip of the 3D volume cut through the $x-z(y=0)$ plane. On the bottom, the $2 \mathrm{D} x-z$ slice plane taken from the 3D simulation. 

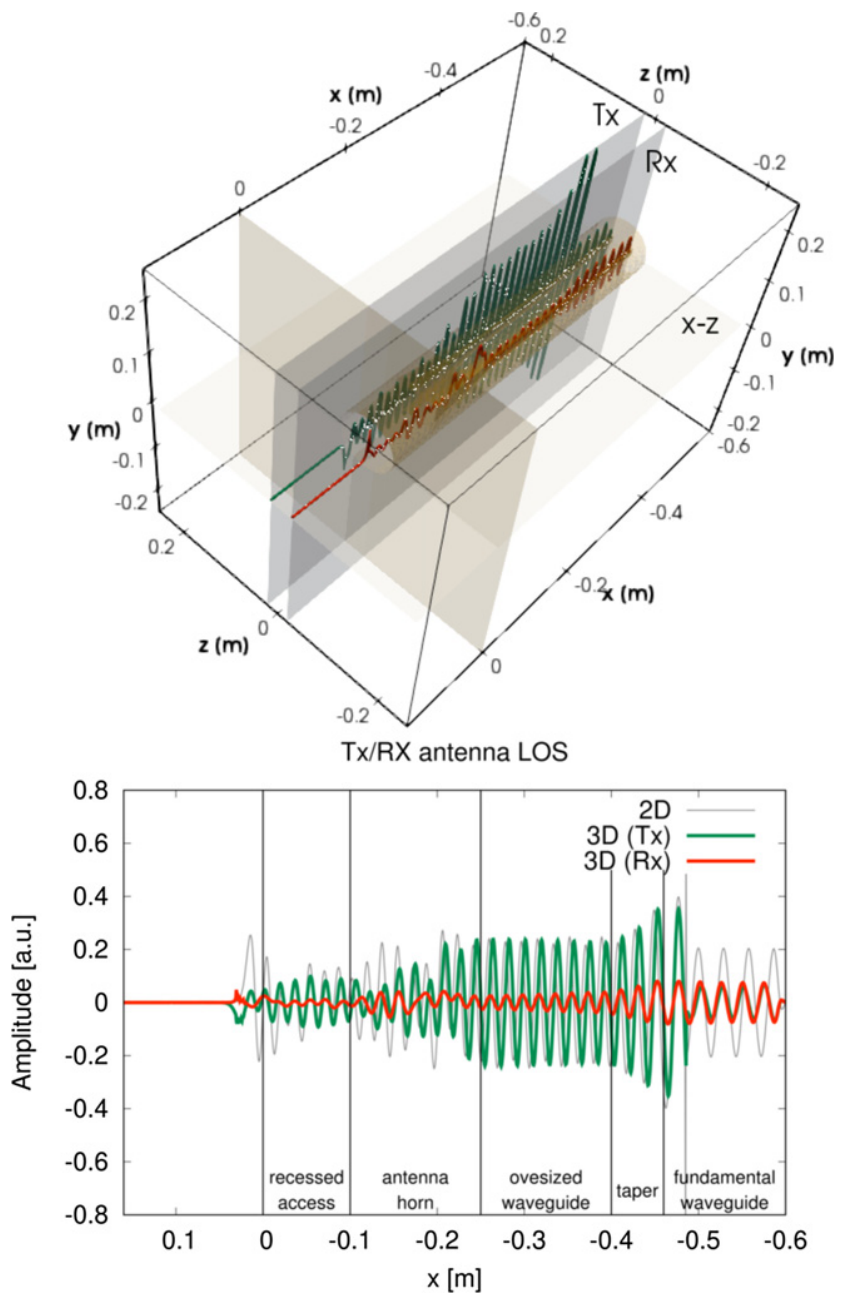

Figure 8. Comparison between 3D and 2D amplitudes of the electric field. On the top, the 3D volume showing the electric field amplitude along the LOSs marked. On the bottom, the relation of amplitudes along the TX and Rx LOSs is displayed together with the 2D result.

\section{Conclusions}

One of the major differences between $2 \mathrm{D}$ and $3 \mathrm{D}$ simulations is the returned values for the amplitude which are more realistic for the latter case, consistent with a decay $\propto R^{-1}$ for 3D and $\propto(\log R \sqrt{ } R)^{-1}$ for 2D. The phase is more resilient to the drop of the third dimension in the present simulations, nevertheless, assessing the true values of amplitude is of major importance to have a proper signal-to-noise ratio $(\mathrm{S} / \mathrm{N})$. In a experimental setup a low value of $\mathrm{S} / \mathrm{N}$ ratio can impair the signal detection, especially on conditions of high turbulence. An accurate knowledge of the amplitude is also important to simulate and implement advanced methods for profile initialisation [6]. Whenever a 2D model is enough, its use allows a much better use of computational resources, as 3D simulations are, as seem before, computationally expensive and should be channelled to the cases where 2D is unable to provide an correct answer, as an aid to help setting up or calibrate a $2 \mathrm{D}$ case study or as a complement to the main $2 \mathrm{D}$ simulations. 


\section{Acknowledgments}

This work has been carried out within the framework of the EUROfusion Consortium and has received funding from the Euratom research and training programme 2014-2018 and 2019-2020 under Grant Agreement No. 633053 and within the framework of the French Federation for Magnetic Fusion Studies (FR-FCM). IST activities also received financial support from Fundação para a Ciência e Tecnologia through project UID/FIS/50010/2019. The views and opinions expressed herein do not necessarily reflect those of the European Commission. Simulations were made using EUROfusion High Performance Computer Marconi, under project REFMULx.

\section{References}

[1] F. da Silva, S. Heuraux, E. Ricardo, A. Silva and T. Ribeiro, Benchmarking 2D against 3D FDTD codes in the assessment of reflectometry performance in fusion devices, 2019 JINST $14 \mathrm{C} 08004$.

[2] F. da Silva et al., Assessment of measurement performance for a low field side IDTT plasma position reflectometry system, Fusion Eng. Des. 168 (2021) 112405.

[3] R. Martone et al., DTT Divertor Tokamak Test facility — Interim Design Report (2019).

[4] L. Frassinetti et al., Global and pedestal confinement and pedestal structure in dimensionless collisionality scans of low-triangularity H-mode plasmas in JET-ILW, Nucl. Fusion 57 (2017) 016012.

[5] F. da Silva, S. Heuraux, S. Hacquin and M. Manso, Unidirectional transparent signal injection in finitedifference time-domain electromagnetic codes - application to reflectometry simulations, J. Comput. Phys. 203 (2005) 467.

[6] R.B. Morales, S. Heuraux, R. Sabot, S. Hacquin and F. Clairet, Reconstruction of hollow areas in density profiles from frequency swept reflectometry, Plasma Sci. Technol. 22 (2020) 064005. 